\title{
Sperm head shaping in ratites: new insights, yet more questions
}

Lizette du Plessis $^{1 *}$ and John T Soley ${ }^{2}$

${ }^{1}$ Electron Microscope Unit, Department of Anatomy and Physiology, Faculty of Veterinary

Science, University of Pretoria, Onderstepoort 0110, South Africa

${ }^{2}$ Department of Anatomy and Physiology, Faculty of Veterinary Science, University of

Pretoria, Onderstepoort 0110, South Africa

Running title: Sperm head shaping in ratites

Keywords: ratites, spermiogenesis, manchette microtubules, acrosome formation, chromatin condensation, perinuclear theca

*Corresponding author: Lizette du Plessis

e-mail address: lizette.duplessis@up.ac.za

\section{Highlights}

- Detailed description of the various factors involved in sperm head shaping.

- First report on the presence of a perinuclear theca (PT) in birds.

- The PT plays a role in stabilizing the sperm head during spermiogenesis in ratites.

- The elaboration of the PT may be used to resolve phylogenetic questions. 


\section{Abstract}

Head shaping in mammalian sperm is regulated by a number of factors including acrosome formation, nuclear condensation and the action of the microtubular manchette. A role has also been suggested for the attendant Sertoli cells and the perinuclear theca (PT). In comparison, relatively little information is available on this topic in birds and the presence of a PT per se has not been described in this vertebrate order. This study revealed that a similar combination of factors contributed to head shaping in the ostrich, emu and rhea, although the Sertoli cells seem to play a limited role in ratites. A fibro-granular structure analogous to the mammalian PT was identified, consisting of sub- and post-acrosomal components. The latter was characterized by stage-specific finger-like projections that appeared to emanate from the cytoplasmic face of the nuclear envelope. They were particularly obvious beneath the base of the acrosome, and closely aligned, but not connected to, the manchette microtubules. During the final stages of chromatin condensation and elongation of the sperm head the projections abruptly disappeared. They appear to play a role in stabilizing the shape of the sperm head during the caudal translocation of the spermatid cytoplasm. 


\section{Introduction}

Formation and shaping of the sperm head has been studied in numerous vertebrate and invertebrate species (Fawcett et al., 1971; Phillips, 1974; Toshimori and Ito, 2003), particularly in mammals, and a link established between defective head formation / structure and compromised fertility (Barth and Oko, 1989; Dadoune, 1995, 2003; Zamboni, 1987; Zhuang et al., 2014). Although conflicting evidence has been presented in the literature, morphological data supplied by conventional transmission electron microscopy, supplemented by the use of animal models, have implicated various cellular components and processes in nuclear (head) morphogenesis (Fawcett, et al., 1971; Hermo et al., 2010; Kierszenbaum et al., 2003, 2004, 2007; Meistrich, 1993). These include the role of the forming acrosome ( Kierszenbaum et al., 2003, 2004, 2007; Russell et al., 1991) inherent properties of the chromatin condensation process (Fawcett et al., 1971; Prisant et al., 2007; Nozawa et al., 2014; Zhuang et al., 2014) and the involvement of the manchette microtubules (Kierszenbaum, 2002; Meistrich, 1993; Meistrich et al., 1990; Nozawa et al., 2014; Russell et al., 1991, 1994; Toshimori and Ito, 2003). In addition, it has been proposed that the ectoplasmic specializations of the Sertoli cells surrounding the sperm head are instrumental in effecting remodeling of the spermatid nucleus (Kierszenbaum et al., 2007; Russell et al., 1994). The interactive nature of the above factors has also been noted (Tovich et al., 2004).

Recent studies based primarily on the immunocytochemical localization of a range of proteins have identified two key components involved in mammalian head morphogenesis, namely, the perinuclear theca (PT) (Mújica et al., 2003; Toshimori and Ito, 2003) and acroplaxome (Kierszenbaum et al., 2003, 2007; Toshimori and Ito, 2003). The PT has been described as the major cytoskeletal component of the sperm head and is credited with binding of the acrosome to the nucleus (Oko, 1995; Oko and Sutovsky, 2009) as well as maintaining 
sperm head shape by acting as a mechanical scaffold (Escalier, 1990; Mújica et al., 2003). The acroplaxome, effectively representing the sub-acrosomal component of the PT (Kierszenbaum et al., 2003) described in other mammals, serves to anchor the acrosome to the nuclear envelope and also guides nuclear elongation (through the interaction of the acroplaxome-manchette perinuclear rings) by providing "a mechanical planar scaffold modulating external clutching forces generated by a stack of Sertoli cell F-actin-containing hoops encircling the elongating spermatid nucleus" (Kierszenbaum et al., 2003, 2007).

Compared to the volume and sophistication of the work carried out on sperm head shaping in mammals, surprisingly little information on this phenomenon has been presented in birds. Based on circumstantial morphological evidence, the circular manchette (CM), which manifests during the earlier stages of avian spermiogenesis, has been implicated in effecting nuclear morphogenesis in a number of non-passerine species (Gunawardana and Scott, 1977; Lin and Jones, 1993; McIntosh and Porter, 1967; Okamura and Nishiyama, 1976; Soley, 1996, 1997). The pattern of chromatin condensation (Fawcett et al., 1971), as well as the limited role of the forming acrosome (Soley, 1996, 1997), have also been proposed as factors determining nuclear shaping in birds. The possible involvement of a PT, or indeed the existence of this structure, has not been reported in birds, although the translucent layer beneath the forming acrosome and the peri-nuclear fibro-granular layer posterior to the acrosome (Aire, 2007, 2014; Fawcett et al., 1971) described in some species would suggest the existence of a PT in this vertebrate order. The recent identification of a unique, transient cytoplasmic structure that manifests during spermiogenesis around the post-acrosomal region of the sperm nucleus in ratites (Du Plessis and Soley, 2013) provides compelling evidence for the presence of an analogous entity, at least in ratites. 
This paper documents the morphological events relevant to head shaping that occur during spermiogenesis in three ratite species, the ostrich (Struthio camelus), emu (Dromaius novaehollandiae) and rhea (Rhea americana albisceus). The work supplements previous observations on this topic (Soley, 1994, 1996, 1997) and also reports for the first time on the presence, and stage-specific elaboration, of material analogous to the mammalian PT in the post-acrosomal region of the developing spermatid.

\section{Materials and Methods}

The testes of 10 sexually mature and active emus (Dromaius novaehollandiae) and ostriches (Struthio camelus), respectively, were collected during the breeding season following slaughter at commercial abattoirs. Processed, resin-embedded testes samples of a rhea (Rhea americana albisceus) were kindly supplied by Dr. David M. Phillips.

Small blocks of tissue were removed from the emu and ostrich testes and immediately fixed for 24 hours at $4{ }^{\circ} \mathrm{C}$ in $4 \%$ glutaraldehyde buffered with $0.13 \mathrm{M}$ Millonig's phosphate buffer, $\mathrm{pH}$ 7.4. Samples were post-fixed for 1 hour in $1 \%$ similarly buffered osmium tetroxide and routinely prepared for transmission electron microscopy (TEM) (Soley, 1997; du Plessis and Soley, 2012). Ultrathin sections were viewed in a Philips CM10 transmission electron microscope (Philips Electron Optical Division, Eindhoven, The Netherlands) operated at $80 \mathrm{kV}$. The resin samples of rhea testes had been fixed and processed as previously described (Phillips and Asa, 1989) and were sectioned and stained as indicated above.

In order to establish the sequence of cellular events characterizing sperm head development on a comparative basis, spermatids were grouped into four categories based on nuclear structure (shape and degree of chromatin condensation) as proposed by Gunawardana and 
Scott (1977) and applied to spermiogenesis in the ostrich (Soley, 1994, 1996, 1997). Phase I

spermatids presented with round nuclei; phase II spermatids with irregularly shaped nuclei; phase III spermatids displayed elongated nuclei containing coarse granular chromatin and phase IV spermatids elongated nuclei with dense, homogeneous chromatin (Fig. 1).

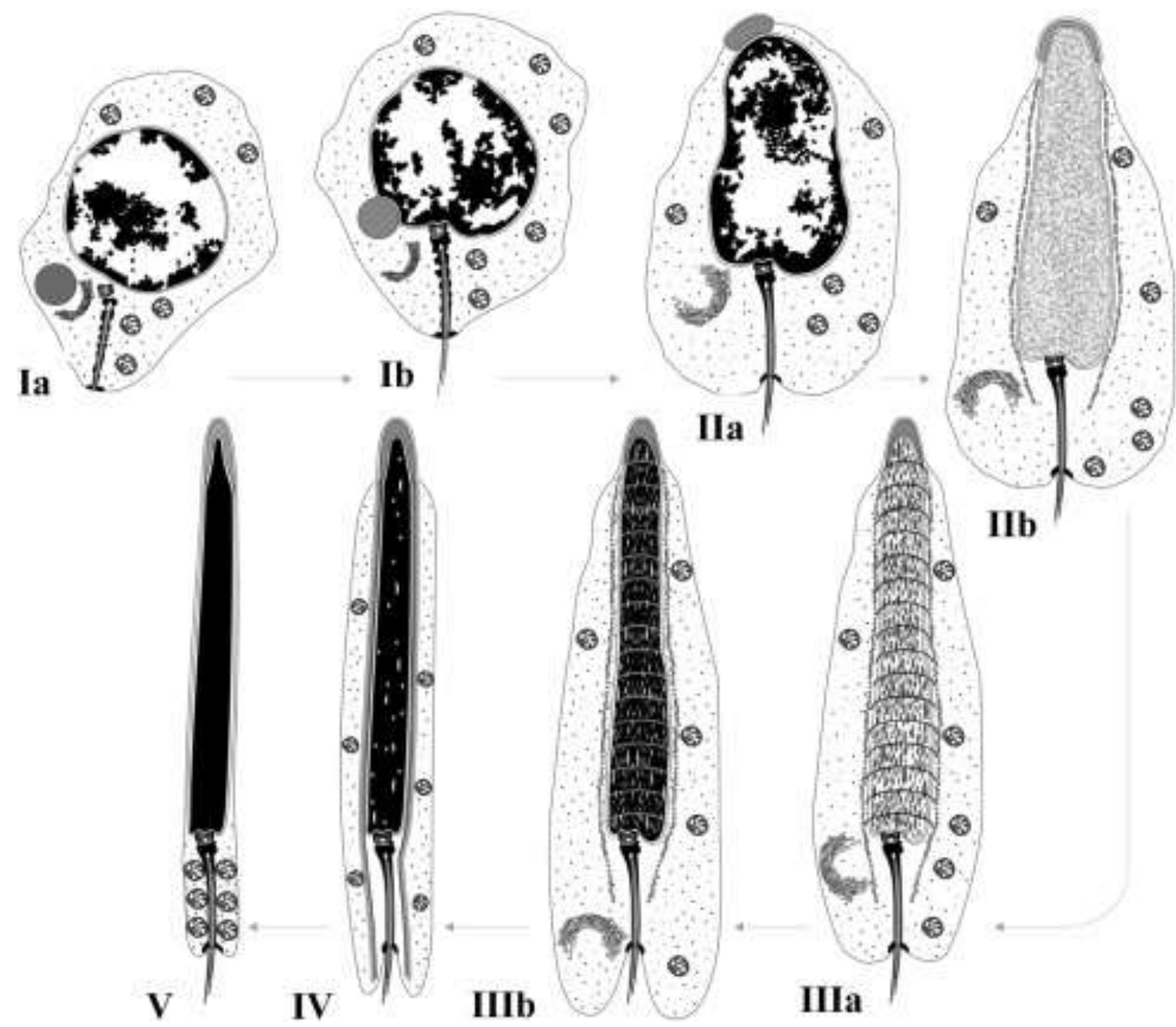

Fig. 1. Schematic illustration of the various phases of spermatid development in the emu. Phase I (Ia \& b): Round nucleus, peripheral aggregations of chromatin, attachment of round acrosomal vesicle and centriolar complex. Phase II (IIa \& b): Irregular shaped nucleus, fine to coarse granular chromatin, poorly organized circular manchette evident. Phase III (IIIa \& b): Elongating nucleus, chromatin more condensed (filamentous) and circular manchette well developed. Phase IV (IV): Elongated nucleus with dense, compacted chromatin, longitudinal manchette present, cytoplasm not yet fully displaced caudally. (V) Fully formed sperm released into lumen of seminiferous tubule. 


\section{Results}

\section{Phase I: Spermatids with round nuclei}

Early round spermatids displayed a prominent, centrally positioned, round nucleus containing scattered clumps of heterochromatin most of which were arranged peripherally against the inner nuclear membrane (Figs. 1-Ia,b, 2a). The intervening clear areas contained fine flocculent material. The cytoplasm displayed numerous mitochondria, as well as smooth and granular endoplasmic reticulum, a Golgi apparatus, chromatoid bodies, the occasional lipid droplet and multivesicular bodies. Most of the organelles were concentrated around the centriolar complex (CC) composed of proximal and distal centrioles. At this early stage, only occasional microtubules were present in the cytoplasm. However, small, regularly spaced deposits of moderately electron-dense granular material appeared close to the outer nuclear membrane in the position later occupied by the microtubules of the CM.

The first obvious sign of acrosome formation was the appearance of typical pro-acrosomal vesicles which coalesced to form a large, round acrosome vesicle filled with homogenous material of moderate electron density. This structure was positioned close to the nuclear membrane in the vicinity of the $\mathrm{CC}$ and was generally associated with the Golgi apparatus.

The acrosomal vesicle subsequently made contact with the outer nuclear membrane, nestling in a shallow concavity formed by the nuclear envelope (Figs. 1-Ib, 2a,b). At the point of attachment the nuclear envelope narrowed and, as a result, this part of the nuclear envelope appeared electron-dense. A thin, translucent layer of cytoplasm remained trapped between the acrosomal vesicle and the nuclear membrane (Fig. 2b). Patches of heterochromatin were always associated with the nuclear aspect of the concavity. In later phase I spermatids the acrosome vesicle was located at a variable distance from the now attached $\mathrm{CC}$, eventually approaching the future cranial pole of the nucleus relative to the forming neck region. The 
acrosome vesicle remained round during this phase of development with signs of collapse (indicated by ruffling of the membrane) towards the end of this phase (Fig. 2b).
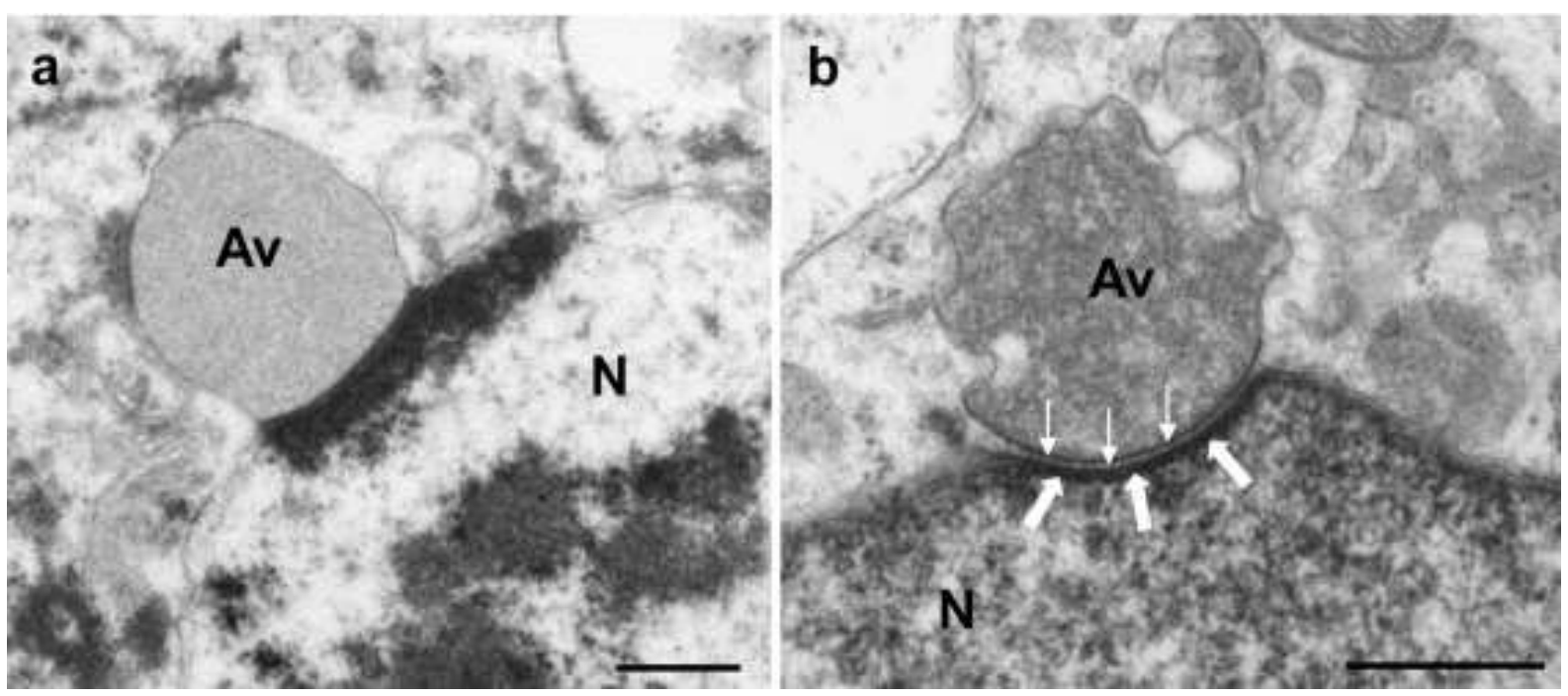

Fig. 2. (a) Phase I emu spermatid. The acrosome vesicle (Av) is attached to the nucleus $(\mathrm{N})$ which displays scattered clumps of heterochromatin. (b) A late phase I ostrich spermatid demonstrating the attached acrosomal vesicle (Av) which shows signs of collapse. Note the translucent later of cytoplasm trapped between the vesicle and the nucleus (thin arrows) and the electron-dense appearance of the adjacent nuclear envelope (arrows). Nucleus $(\mathrm{N})$. Bars $=0.5 \mu \mathrm{m}$.

Based on the above observations it was clear that in phase I spermatids the formation of the acrosome was initiated as well as placement of the $\mathrm{CC}$ and associated development of the flagellum. No obvious signs of head-shaping were apparent although the sub-acrosomal component (the translucent layer) of the PT (see below) was established.

\section{Phase II: Spermatids with irregularly shaped nuclei}

The onset of phase II was characterized by a change in the shape of the nucleus from round to pear-shaped, dumbbell-shaped or scalloped in longitudinal profile (Fig.1-IIa). Associated with this change in nuclear profile was the appearance of circumferentially disposed microtubules in the cytoplasm close to the outer nuclear membrane, and which were present in greater numbers at the points of nuclear constriction (Fig. 3a). Chromatin clumping gradually became less obvious and the nucleus was filled with fine granular material with 
intervening clear spaces (Figs. 3c,d). Changes in the appearance of the chromatin were accompanied by an increase in the number of microtubules located at the nuclear perimeter and which collectively constituted the forming CM. The CM ran the entire length of the nucleus, starting just below the base of the acrosome and ending at the proximal part of the midpiece. In the rhea, the $\mathrm{CM}$ extended anteriorly beyond the base of the acrosome within a cytoplasmic collar (Fig. 3b), a phenomenon only occasionally observed in the ostrich but not in the emu. In transverse sections of phase II spermatids the microtubules appeared as loosely arranged rings wrapped around the nucleus (Fig. 4a), while in longitudinal sections they were visible as a single row of microtubules closely aligned to the outer nuclear membrane (Fig. 3b). The deposits of granular material observed during later phase I spermatids were intimately associated with the manchette microtubules (see Fig. 3a). During subsequent nuclear elongation and condensation the chromatin displayed localized separation from the inner nuclear membrane, leaving prominent gaps between the nuclear envelope and the karyoplasm. This was particularly evident just below the base of the acrosome but never beneath the acrosome itself (Fig. 3b). Also obvious in this region was the accumulation of flocculent material in the narrow zone of cytoplasm between the outer nuclear membrane and the microtubules of the forming CM. Similar material was associated with all parts of the nuclear envelope, including the nuclear base (but not the implantation fossa), constituting a perinuclear tunic surrounding the post-acrosomal portion of the nucleus. 


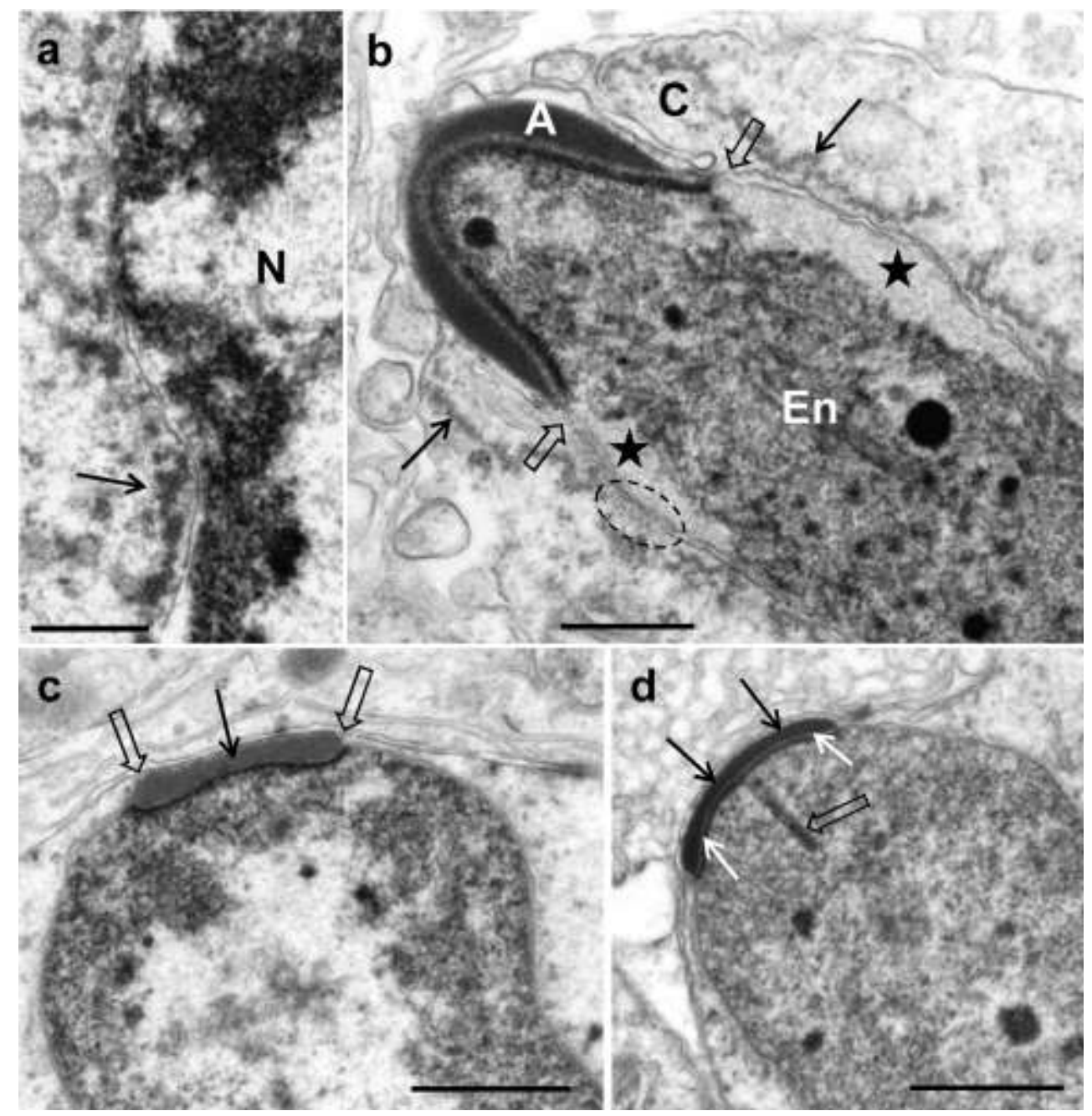

Fig. 3. (a) High magnification of a longitudinal section of a phase II emu spermatid showing a nuclear constriction with an accumulation of microtubules and granular material (arrow) in the adjacent cytoplasm. Nucleus (N). (b) Late phase II rhea spermatid. The main morphological features include a cup-shaped acrosome (A), cytoplasmic collar (C) with a cranial extension of the circular manchette (thin arrows), post-acrosomal perinuclear theca (block arrows) showing localized concentration of finger-like elaborations (dashed oval) and shrinkage of the chromatin away from the inner nuclear membrane (stars). Endonuclear canal with perforatorium (En). (c) Phase II ostrich spermatid displaying the typical dumb-bell-shaped forming acrosome (thin arrow). The spermatid plasmalemma has been drawn over the acrosome (block arrows). (d) Phase II rhea spermatid displaying a crescent-shaped acrosome (thin arrows), translucent sub-acrosomal layer (white arrows) and the forming endonuclear canal (block arrow). Bars $=0.5 \mu \mathrm{m}$.

Subsequently, isolated, moderately electron-dense groups of finger-like projections made their appearance in the perinuclear tunic. The base of the projections was closely associated with the outer leaflet of the nuclear membrane, appearing to emanate from its cytoplasmic surface, whereas the apex stretched close to, but did not make contact with, the manchette microtubules. In more mature phase II spermatids (typically displaying a greater degree of shrinkage of the chromatin away from the nuclear envelope, and a more organized CM), the 
projections were arranged in variably sized groups (Fig. 3b), arbitrarily situated at any point along the nuclear perimeter, with the exception of the implantation fossa which was occupied by the proximal centriole and elements of the connecting piece. They were particularly obvious around the nuclear shoulder abutting the base of the forming acrosome.

During phase II the acrosome vesicle, firmly attached to the nuclear envelope, occupied the opposite pole of the nucleus relative to the CC. The round vesicle typical of phase I spermatids was observed to collapse onto the nucleus, flatten to form a compressed dumbbell-shaped structure (Fig. 3c), and spread over the tip of the now elongating nucleus as the forming acrosome, eventually taking on a crescent shape (Fig. 3d). The original concave nuclear depression housing the acrosome vesicle now formed a blunt convex projection beneath the vesicle which appeared more electron-dense due to the compaction of its contents. Progressive caudal extension of the forming acrosome produced a cap-like structure which coincided with gradual narrowing of the nuclear apex (Fig. 3b). The translucent layer between the forming acrosome and the nuclear envelope was more obvious and displayed a collection of flocculent material. This material was observed to be continuous with the perinuclear material (theca) surrounding the post-acrosomal region of the nucleus. The modified nuclear envelope beneath the forming acrosome was further accentuated by the presence of a thin deposit of karyoplasm along its nuclear aspect. In the ostrich and rhea, a localized caudal extension of the modified nuclear envelope into the karyoplasm was evident at this stage, resulting in the formation of a narrow endonuclear canal which communicated with the translucent layer located beneath the acrosome (Fig. 3d). The canal was filled with material similar to that observed in the translucent layer and constituted the perforatorium (acrosomal rod). Based on the observation that the perforatorium does not appear to play a role in nuclear shaping in ratites (Soley, 1996), further development of this structure is not 
addressed in the present study. The plasmalemma was generally closely applied to the forming acrosome (Fig. 3c), in some instances accompanied by a hemi-desmosome-like junction or rudimentary adhering junction related to the point of contact with the surrounding Sertoli cell cytoplasm. Elements of the Sertoli cell endoplasmic reticulum were closely associated with the hemi-desmosomes. However, as noted above, posterior expansion of the acrosome was accompanied by the formation of a cytoplasmic collar encircling, but separated from, the forming acrosome and which contained microtubules of the CM. Progressive development of the forming acrosome coincided with gradual narrowing of the nuclear apex. The Sertoli cell cytoplasm surrounding the acrosome manifested as a solid mass or as isolated projections. In some instances the cytoplasmic collar of the spermatid was separated from the acrosome by a mantel of Sertoli cell cytoplasm (see Fig. 6a of a later phase spermatid). Profiles of smooth endoplasmic reticulum were associated with the acrosomal surface of the Sertoli cell but no filamentous elements were observed.

During phase II the tip of the nucleus appears to be molded by the gradual transformation in shape of the acrosomal vesicle. Localized concentrations of the CM microtubules deform the round nucleus, narrowing and lengthening it. The chromatin independently shrinks away from the nuclear envelope, gradually becoming more compacted. The post-acrosomal PT is established, maintains continuity with the sub-acrosomal component, and develops elaborations in the form of finger-like extensions.

\section{Phase III: Spermatids with elongated nuclei containing coarse granular / filamentous} chromatin

The nucleus continued to elongate during this phase accompanied by further condensation and compaction of the chromatin. Further coalescing of the granules observed in phase II 
spermatids resulted in the formation of larger electron-dense granules (Fig. 5) which in turn condensed to form longitudinally disposed filaments separated by clear spaces (Figs. 1-IIIa,b, 4b). Concomitant with these changes, the gap between the chromatin and inner nuclear membrane widened and was prominent along much of the length of the nucleus (Figs. 4b, 5). The well-developed CM presented as a single row of closely aligned microtubules in longitudinally sectioned spermatids (Fig. 5) supported periodically by additional groups of microtubules situated on the cytoplasmic aspect of the manchette. In transverse sections of the nucleus the manchette appeared as a single, sometimes overlapping, microtubular coil or coils, depending on where the manchette was sectioned (Figs. 4b,c). Neighboring microtubules were connected by short filamentous linkers but no connection with the nuclear envelope was obvious. The finger-like projections associated with the outer nuclear membrane increased in number and prominence, forming small scattered pockets throughout the surface of the nucleus. The morphological characteristics and spatial orientation of the projections remained unchanged.

The manchette microtubules lost their concentric arrangement in later phase III spermatids and became randomly oriented around the nucleus, in some sections appearing to have dispersed. During this transitional stage regularly spaced units of moderately electron-dense material appeared in the vicinity of the disbanding manchette microtubules (Fig. 4c). Towards the end of phase III, a second set of microtubules, the longitudinal manchette (LM), orientated longitudinally and parallel to the nucleus, appeared, concomitant with the gradual disappearance of the blocks of electron-dense material. The LM extended from the base of the acrosome to well beyond the annulus. The chromatin appeared more compacted but remained fibrillar in nature and there was a gradual and continual caudal migration and reduction of the spermatid cytoplasm. 


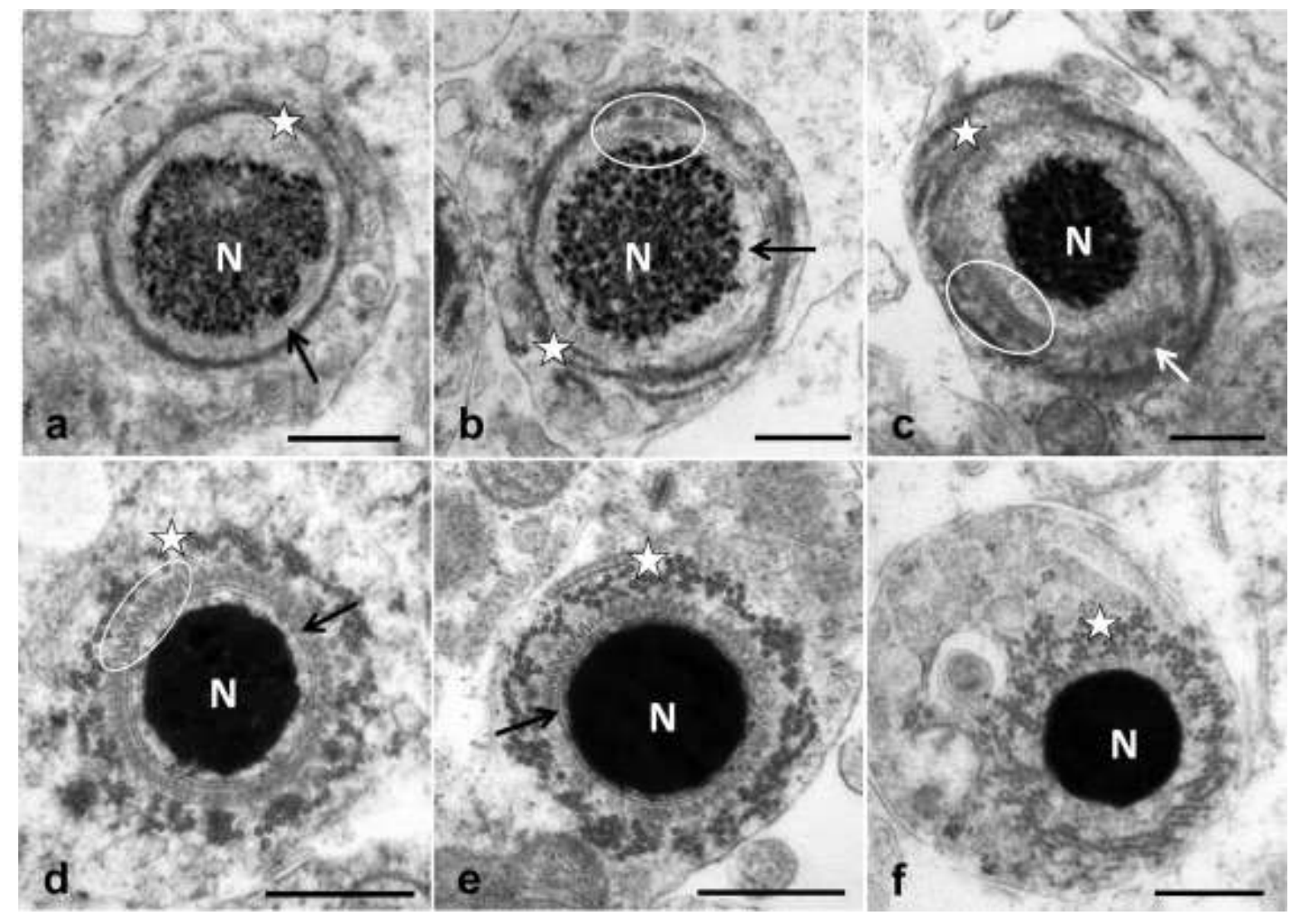

Fig. 4. Transverse sections through the head region of developing emu spermatids illustrating the relationship between chromatin condensation, manchette microtubules and finger-like projections of the post-acrosomal perinuclear theca. (a) Phase II spermatid with the forming circular manchette (star). Note the fine granular chromatin in the nucleus $(\mathrm{N})$ and the zone of flocculant material (arrow) between the manchette and the nuclear envelope. No projections are visible. (b) Early phase III spermatid with coarse chromatin granules and an obvious gap (arrow) between the condensing chromatin and inner nuclear membrane. Isolated pockets of projections (encircled) make their appearance in the perinuclear theca. (c) Phase III spermatid with fibrillar chromatin, disbanding circular manchette (star), distinctive finger-like projections (encircled) and blocks of moderately electron-dense material (white arrow). (d) Early phase IV spermatid displaying the longitudinal manchette (star). The chromatin is highly condensed and the projections (encircled) within the perinuclear theca appear to be associated with the cytoplasmic face of the outer nuclear membrane (arrow). A relatively narrower gap persists between the condensing chromatin and the nuclear envelope. (e) Phase IV spermatid with welldeveloped longitudinal manchette (star) and condensed chromatin. The perinuclear theca projections are visible around the entire nuclear envelope (arrow) which is closely applied to the condensed chromatin. (f) An advanced phase IV spermatid with the longitudinal manchette (star) still in evidence. The perinuclear theca projections are no longer present. Nucleus $(\mathrm{N})$. Bars $=0.5 \mu \mathrm{m}$.

The membrane-bound acrosome, covered only by the plasmalemma but closely associated with Sertoli cell cytoplasm, continued to extend caudally around the tip of the nucleus. It was initially cup-shaped but progressively narrowed and lengthened to form a rounded cone (Fig.

5). The walls of the forming acrosome were of equal width although they narrowed and flared outwards at the base to accommodate the forming nuclear shoulder (Fig. 5). The contents of the acrosome remained relatively electron-dense. Extension and narrowing of the 
developing acrosome was accompanied by constriction of the apical aspect of the nucleus

which was transformed into a blunt cylinder (the future nuclear rostrum) continuous with the rest of the nucleus where it sloped and widened to form the nuclear shoulder. The perimeter of the nuclear rostrum remained clearly demarcated by narrowing of the nuclear envelope (Fig. 5). The sub-acrosomal component of the PT formed a prominent layer of material which, as in the previous phase, was continuous with the post-acrosomal elements of the theca. The rapid expansion of the acrosome appeared to be responsible for the deepening of the cytoplasmic collar observed in phase II spermatids. Although interlinked microtubules of the $\mathrm{CM}$ were present in the collar, no finger-like projections were ever observed (Fig. 5).

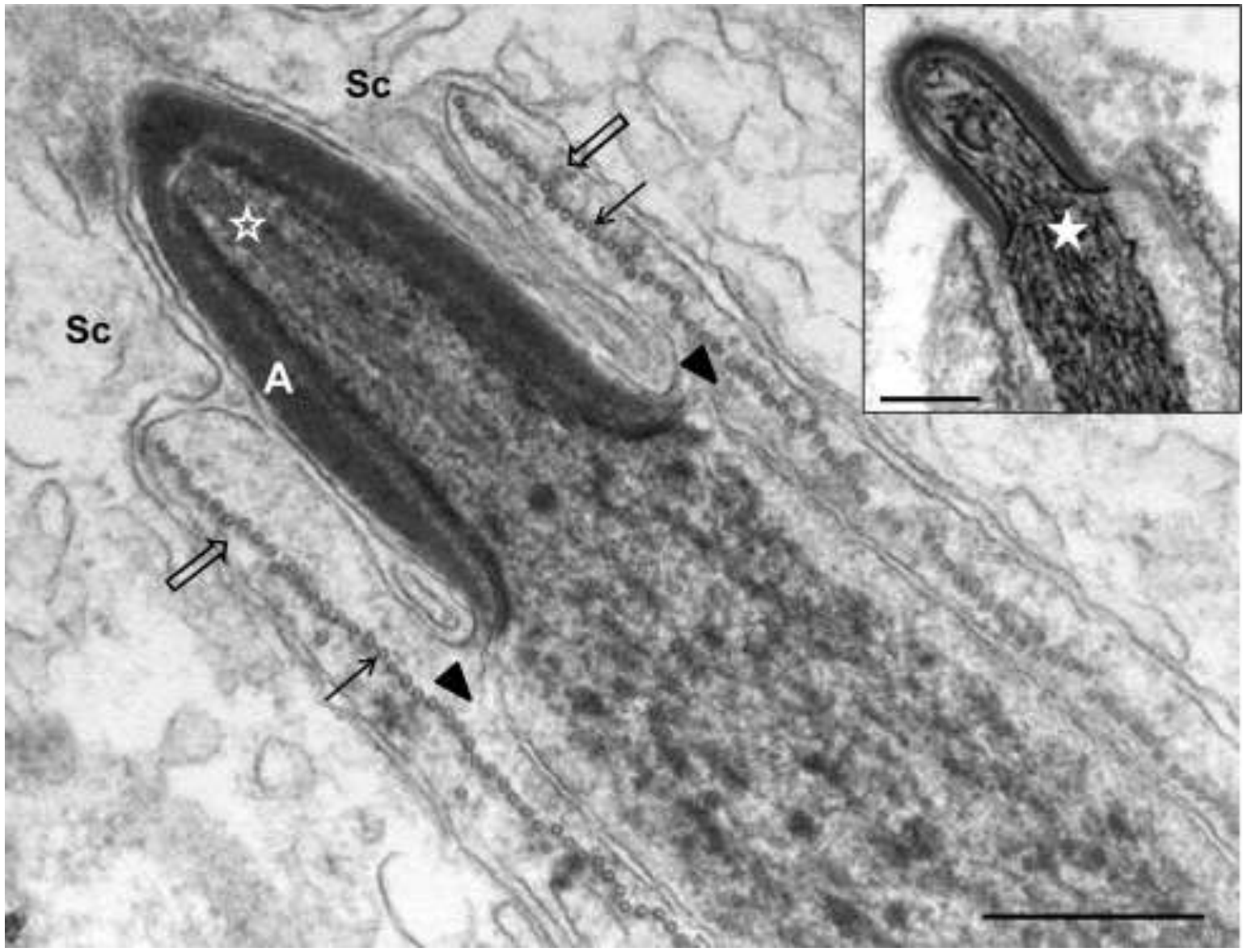

Fig. 5. Early phase III rhea spermatid showing the forming cone-shaped acrosome (A) surrounded by a cytoplasmic collar (arrows) containing elements of the circular manchette (thin arrows). Note the endonuclear canal and associated acrosomal rod (star), and fibrillar nature of the karyoplasm. The post-acrosomal perinuclear theca (arrowheads) shows no obvious elaborations. Sertoli cell cytoplasm (Sc). Inset. A slightly later phase III emu spermatid characterized by the advanced fibrillar nature of the condensing chromatin (star). Note the absence of a cytoplasmic collar and endonuclear canal. Bars $=0.5 \mu \mathrm{m}$. 
Continued development of the acrosome during phase III results in the formation of the nuclear rostrum. The definitive $\mathrm{CM}$ accompanies the elongating and condensing nucleus before transforming into the LM. The fibrillar karyoplasm remains isolated from the inner nuclear membrane while the finger-like elaborations continue to develop in the postacrosomal PT.

\section{Phase IV: Spermatids with elongated nuclei and homogeneous, electron dense} chromatin

Phase IV spermatids displayed a long, narrow nucleus filled with homogeneous, electrondense chromatin (Fig.1-IV). Areas of incomplete chromatin condensation were occasionally observed, particularly towards the nuclear base. The gap between the condensing chromatin and the inner nuclear membrane had narrowed appreciably (Figs. 4d, 6a) and disappeared in late stage phase IV spermatids leaving the nuclear envelope tightly applied to the karyoplasm (Figs. 4e,f). The blunt, rounded tip of the nuclear rostrum seen during phase III was transformed into a tapered point, covered by the acrosome. The dense nature of the nuclear envelope covering the rostrum was no longer obvious due to the complete condensation of the chromatin. There was a marked reduction in the amount of cytoplasm surrounding the nucleus, with only the microtubules of the LM and the occasional mitochondrion being observed in this region (Fig.1-IV).

The LM developed maximally during this phase and consisted, in transverse section, of approximately three to four ill-defined rows of microtubules (Figs. 4d-f) interconnected by short filamentous linkers. The inner row of microtubules was poorly represented, consisting of only a limited number of individual microtubules situated in close proximity to the outer 


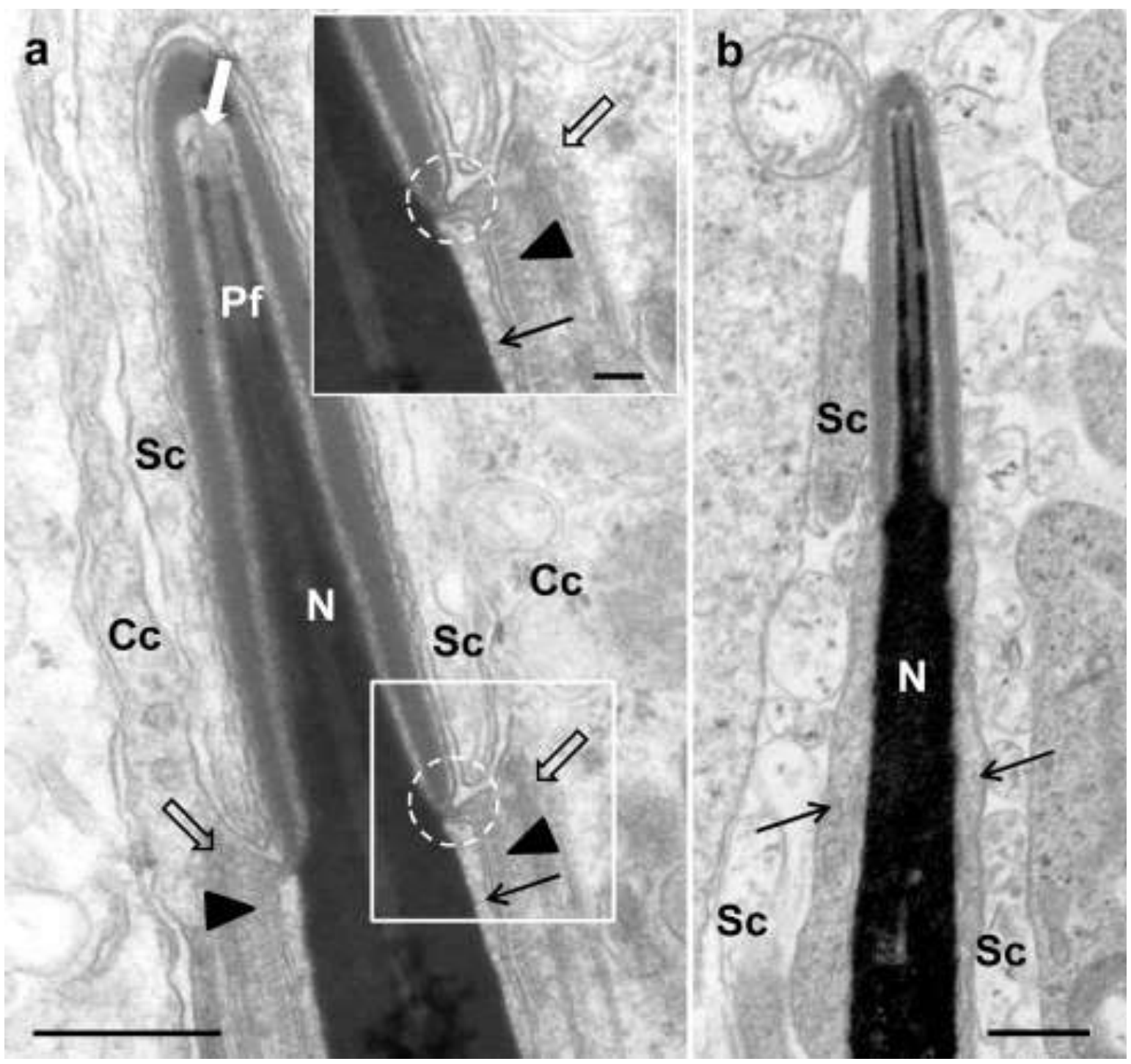

Fig. 6. Phase IV ostrich spermatid. (a) This cell still displays a cytoplasmic collar (Cc) but which is devoid of microtubules. The longitudinal manchette originates in the cytoplasm at the base of the acrosome (block arrows), the post-acrosomal perinuclear theca displays finger-like projections (arrowheads) and a narrow gap persists between the condensing chromatin and the nuclear envelope (thin arrows). Note the continuity between the sub-acrosomal and post-acrosomal components of the perinuclear theca (encircled) as well as that between the sub-acrosomal material and the perforatorium (white arrow). Sertoli cell cytoplasm (Sc), Perforatorium (Pf), Nucleus (N). Inset. Enlargement of the area blocked in the micrograph. (b) A more advanced phase IV ostrich spermatid. The cytoplasmic collar has retreated distally and the residual cytoplasm concentrated around the body of the nucleus (thin arrows) is devoid of manchette microtubules and the finger-like elaborations of the PAS. Sertoli cell cytoplasm (Sc). Bars $=0.5 \mu \mathrm{m}$; inset bar $=0.1 \mu \mathrm{m}$.

nuclear membrane-associated projections that were prominent at this stage of development (see below). In longitudinal section the manchette displayed two to four rows of longitudinally disposed microtubules (Fig. 6). This difference in the number of rows seen in longitudinal profile could be ascribed to the small probability of sectioning the poorly represented inner row of microtubules. As previously noted in late phase III spermatids, the 
microtubules of the LM extended from the base of the acrosome to the caudal end of the midpiece, and often beyond the annulus to within the cytoplasm surrounding the flagellar canal (Fig.1-IV). Towards the end of this phase, following the disappearance of the outer nuclear membrane-associated projections (see below) and just prior to the mature sperm being released into the lumen of the seminiferous tubule, the manchette microtubules abruptly disappeared (Fig. 4f). The zone of cytoplasm housing the post-acrosomal PT was thus temporarily expanded following the loss of the microtubules after which it narrowed progressively as the cell matured (Fig. 6b).

The projections associated with the nuclear envelope lodged within the post-acrosomal PT were well-developed and increased markedly in number. They formed a uniformly and evenly-spaced array which appeared to cover the entire surface of the nucleus (Figs. 4d,e), although they were more prominent in the region immediately beneath the base of the acrosome. The morphological characteristics of the definitive form of the projections have previously been described in detail (Du Plessis and Soley, 2013). The mean length of the projections was $29.8 \pm 4.2 \mathrm{~nm}$ with a mean width at the base of $13.4 \pm 2.5 \mathrm{~nm}$. Although the base of the projections appeared to be in contact with the cytoplasmic leaf of the nuclear envelope, preliminary electron tomography results indicated that both structures were independent (Du Plessis, 2013). The mean distance between projections was $19.6 \pm 4.4 \mathrm{~nm}$. In late stage IV spermatids the array of projections completely disappeared leaving a zone of fine, moderately electron-dense particulate matter between the outer nuclear membrane and the manchette microtubules (Fig.4f). This transition was abrupt and no intermediate stage was observed in the material studied. 
The acrosome reached its definitive form during this phase, manifesting as a blunt cone covering the tapered tip (rostrum) of the nucleus. The wall of the acrosome was widest at the nuclear apex and thinned progressively towards its termination at the nuclear shoulder (Fig. 6). The acrosomal contents appeared less electron-dense than during the previous phase presumably due to the larger area occupied. The sub-acrosomal space containing the subacrosomal component of the PT was particularly obvious (Fig. 6).

Mature sperm displayed the typical head morphology previously reported in the ostrich (Soley, 1993) and emu (Du Plessis and Soley, 2014). The sub-acrosomal component of the PT was always obvious, revealing the fine flocculent moderately electron-dense material observed in the developing spermatids. In contrast, the post-acrosomal PT was difficult to resolve (except occasionally near the base of the nucleus) due to the close apposition of the plasmalemma to the nucleus.

\section{Discussion}

Sperm head shaping in birds remains a controversial topic and various opinions have been expressed in the literature regarding the factors involved in this process. Previous studies on spermiogenesis in non-passerine birds have implicated the circular manchette in nuclear shaping (Lin and Jones, 1993; McIntosh and Porter, 1967; Nagano, 1962; Okamura and Nishiyama, 1976; Soley, 1997), as well as the possible role of the chromatin condensation process (Fawcett et al., 1971; Soley, 1997). However, the present comparative investigation suggests that sperm head shaping in ratites is a multifactorial phenomenon, involving the forming acrosome and PT in addition to the factors previously proposed. The various processes that appear to influence head shaping are closely co-ordinated during the early stages of spermiogenesis. 


\subsection{The role of the manchette microtubules}

The manchette is a transient cytoplasmic structure composed of an array of microtubules that is associated with spermiogenesis in most, but not all, vertebrate species. When present, notable differences in the appearance and orientation of the manchette between mammals and non-passerine birds are apparent. Most mammalian spermatids typically only display a LM with its point of attachment at the nuclear ring, a sub-plasmalemmal density situated just beneath the base of the acrosome (Barth and Oko, 1989; Fawcett et al., 1971; Holstein and Roosen-Runge, 1981; Ploën, 1971). In contrast, spermatids of a number of avian orders characteristically display both a CM and a LM (Aire, 2014), although the latter is not associated with a nuclear ring. A transitional stage between the two manchettes has been described in some avian species such as the ostrich (Soley, 1997), rhea (Phillips and Asa, 1989), turkey (Aire, 2003) and African collared dove (Mattei et al., 1972).

In ratites, the first obvious morphological indication of sperm head shaping is the deformation of the round nucleus of Phase I spermatids, seemingly by the forming CM. The multiple sites of nuclear constriction characterizing this process in Phase II spermatids are marked by a concentration of circularly disposed microtubules as previously described for the ostrich (Soley, 1997) and various other non-passerine species (Aire, 2014). The cumulative effect of this process transforms the nucleus into a scalloped, elongated structure that eventually manifests as a long, narrow cylinder. Initial formation of the CM and localized distortion of the nuclear envelope takes place in advance of any overt signs of chromatin condensation (see below). Additional evidence has been presented in the literature supporting the role of the CM in effecting nuclear shaping in birds. As summarized by Aire (2007, 2014), the round nucleus of Step 4 spermatids (based on the steps of spermiogenesis 
described in the turkey (Aire, 2003)) in non-passerine birds is surrounded by small groups of microtubules positioned close to the outer nuclear membrane, particularly around the rostral aspect. During the next stage (Step 5) the nucleus becomes pear-shaped (Aire, 2007) or teardrop shaped (Lovas et al., 2012). This would appear to indicate that the rostral concentration of microtubules is instrumental in re-modelling that part of the nucleus, in contrast to the caudal part which remains rounded. However, the anterior-posterior gradient observed during the chromatin condensation process in some species (Zhuang et al., 2014) may also account for this phenomenon (see below). The spatial arrangement and extent of the two sets of manchette microtubules would further seem to indicate a role for the $\mathrm{CM}$ in head shaping in ratites. When fully developed the CM terminates around the proximal part of the distal centriole (essentially coiled around and restricted to the nucleus), whereas the LM, implicated in the caudal translocation of spermatid cytoplasm in mammals (Sperry, 2012; Toshimori and Ito, 2003), may reach well beyond the annulus.

Although these observations present compelling evidence for the role of the $\mathrm{CM}$ in avian sperm head shaping, conflicting opinions have been expressed in this regard. Fawcett et al. (1971), based on observations of spermiogenesis in the pigeon, were of the opinion that the single row of microtubules forming the $\mathrm{CM}$ in this species would be unable to generate the constrictive force required to deform the nucleus. In contrast, McIntosh and Porter (1967), in a detailed study of spermiogenesis in the domestic fowl, concluded that the CM was "a helical system of microtubules [that] could give rise to forces which would both constrict the diameter of the nucleus and guide its elongation". The overlapping nature and linkage of individual microtubular coils described in the fowl (McIntosh and Porter, 1967) were also observed in the ratites studied, again strongly supporting the constrictive potential of the $\mathrm{CM}$ in re-modelling the nucleus, at least during the early stages of spermiogenesis. 
Further complicating the proposed head-shaping role of the CM is the observation that nuclear elongation proceeds in some birds without the presence of this structure. It is reportedly absent, for example, in the common swift (Jamieson and Tripepi, 2005), alpine swift and nightjar (Tripepi et al., 1991), and in the wattled jacana (Saita et al., 1983), although a perinuclear row of longitudinally disposed microtubules is observed in these species with (as in the swifts), or without (as in the night-jar and jacana), an attendant collection of similarly disposed microtubules in the surrounding Sertoli cells. Moreover, based on the paucity of cytoplasmic microtubules observed during spermiogenesis, no microtubular manchette is deemed to be present in the cuckoo (Saita et al., 1982). Similar findings have been reported in various vertebrate and invertebrate species (Phillips and Asa, 1989) suggesting that other factors may be responsible for head shaping in these species. However, disruption or incorrect alignment of the manchette microtubules in birds (McIntosh and Porter, 1967) and mammals (Meistrich et al., 1990; Nozawa et al., 2014; Russell et al., 1991, 1994) displaying this structure results in the formation of abnormally shaped heads, which again points to the possible influence of the manchette on sperm nuclear shaping. The vital contribution of the manchette microtubules to the acrosome-acroplaxome-manchette complex (see below) is illustrated by the fact that removal of the novel manchette-associated protein, Fused, results in abnormal head-shaping and tail morphogenesis (Nozawa et al., 2014).

The LM in ratites closely resembles that of mammals in respect of its composition and orientation. However, it is not attached anteriorly to the plasmalemma by a nuclear ring as in mammals and only appears when sperm head elongation and chromatin condensation are well-advanced. By virtue of its role in the acrosome-acroplaxome-manchette complex (see below), the mammalian LM is viewed as an essential component in the assembly of the 
sperm tail, shaping of the sperm head and chromatin condensation (Kierszenbaum, 2002). In ratites the LM is of relatively short duration. Its existence coincides with the caudal displacement of excess cytoplasm to the base of the head/midpiece suggesting that it assists in channeling the residual cytoplasm. The finger-like elaborations of the post-acrosomal component (PAS) of the PT are best developed at this stage, particularly in the region beneath the base of the acrosome (see below). Whether this represents an "attachment site" similar to that of the mammalian nuclear ring remains unknown. It is also conceivable that the LM with its interlinked microtubules and peri-nuclear location assists in maintaining nuclear shape during modification of the cytoplasm. The elaborations, which are found throughout the PAS at this stage of development and retain their close relationship with the manchette microtubules and the outer nuclear membrane, would appear to assist in this respect.

\subsection{Chromatin condensation}

While rejecting the role of the manchette microtubules, Fawcett et al. (1971) proposed that sperm head shape "may be largely determined from within by a specific genetically controlled pattern of aggregation of DNA and protein during condensation of the chromatin". More recent evidence would appear to support this hypothesis. A correlation has been demonstrated between aberrant chromatin condensation and sperm head shape. In man, for example, a high percentage of abnormally elongated heads display altered chromatin compaction (Prisant et al., 2007), whereas CHD5 (a chromatin remodeling nuclear protein) deficient mice display abnormal head morphology in elongating spermatids and epididymal spermatozoa (Zhuang et al., 2014). Moreover, in some animal species condensation of the chromatin occurs without the presence of a manchette (Phillips and Asa, 1989). 
In birds, decondensation of the nuclear chromatin of early spermatids leads to the karyoplasm taking on the form of homogeneously distributed, finely granular material (Aire 2007, 2014). A similar sequence of changes to the nuclear chromatin is also observed in ratites. Shortly after the $\mathrm{CM}$ is established in ratites the chromatin begins to condense forming a more compact fibrillar mass. In late phase II spermatids, the condensing chromatin, excluding the contents of the nuclear rostrum beneath the developing acrosome (see below), begins to shrink away from the surrounding nuclear envelope. This process is most conspicuous immediately beneath the forming nuclear rostrum, possibly indicating that chromatin condensation occurs in an anterior-posterior direction as reported, with the exception of man, in mammalian sperm (Dadounne, 1995; Zhuang et al., 2014). As noted above, compaction and shrinkage of the chromatin only occurs well after the appearance of the CM and initial elongation of the nucleus. The resulting gap between the condensing chromatin and the inner nuclear membrane remains evident during most stages of the condensation process although a substantial degree of constriction of the nuclear envelope, seemingly by the microtubules of the enveloping CM, is apparent. This phenomenon has not specifically been described in other non-passerine birds (Aire 2007, 2014), although micrographs of early spermatids in some avian species appear to show a similar contraction of the karyoplasm (see Lovas et al., 2012). Based on these observations in ratites it would appear that the $\mathrm{CM}$ is responsible for the initial deformation and subsequent elongation of the spermatid nucleus after which the chromatin condenses independently within the confines of the nuclear envelope. A similar conclusion was reached by Russell et al. (1991) in a study of rodent spermiogenesis where it was noted that "chromatin condensation primarily reinforces the shape changes already brought about by the manchette and other structural elements acting on the nucleus". To what extent the formation of the longitudinally oriented chromatin fibrils that characterize the 
condensation process in ratites is intrinsic in nature, or the result of external modelling forces, remains undetermined.

\subsection{Role of the forming acrosome}

As observed in other vertebrates, an intimate relationship is obvious between the forming acrosome and nucleus during the early stages of spermiogenesis in ratites. The point of contact between the acrosomal vesicle (also referred to as the acrosome granule [Aire, 2007]) and the nucleus is marked by the formation of a nuclear depression and modification of the nuclear envelope which adopts a regular appearance. Evidence exists in the mammalian literature suggesting that the modified nuclear envelope is instrumental in guiding the spread of the developing acrosome and positioning of the nuclear ring which determines the location of the LM (Russell et al., 1991). In ratites, following the collapse and gradual spreading of the acrosomal vesicle, the apex of the now visibly elongating nucleus becomes bluntly rounded and narrows to eventually form the tapered nuclear rostrum. During the remodeling of the nuclear apex, the forming acrosome remains closely bound to the modified nuclear envelope but separated from it by a layer of flocculent material which represents the subacrosomal component of the PT (see below).

The influence of the forming acrosome on shaping the apex of the nucleus is further supported by the observation that the condensing chromatin of the nuclear rostrum remains firmly aligned with the encompassing nuclear envelope and does not shrink away as occurs in other parts of the nucleus, particularly in the region just beneath the base of the acrosome. Additionally, in the rhea and to a lesser degree in the ostrich, the acrosome appears to develop more rapidly than the caudal retraction of the spermatid cytoplasm, actively pushing 
between the nuclear apex and the surrounding spermatid cytoplasm to form a cytoplasmic collar lined by the plasmalemma.

Alternatively, it has been suggested that the forming nuclear rostrum guides the shaping of the acrosome. This would appear unlikely in non-passerines as a conical acrosome is formed in a number of species without the presence of a nuclear rostrum (Humphreys, 1975; Jamieson, 2007; Jamieson et al., 1995; Lovas et al., 2012; Phillips et al., 1987; Saita et al., 1982; Samour et al., 1986), although a well-developed perforatorium may fulfill this role. In these birds the acrosome ends adjacent to the nucleus and does not overlap it. A nuclear rostrum is not formed and the nuclear diameter remains the same throughout its length, again indicating the potentially compressive or modeling effect of the forming acrosome. In contrast to its apparently limited role in non-passerine birds, the mammalian acrosome is widely reported to play a significant part in sperm head shaping as part of a complex mechanism involving various intrinsic and extrinsic factors and is addressed below.

\subsection{The perinuclear theca (PT)}

During spermiogenesis the PT manifests as a continuous layer of detergent resistant material that covers the entire surface of the nucleus with the exception of the implantation fossa and can be subdivided into a sub-acrosomal layer (SAL) and a post-acrosomal sheath (PAS) or calyx (Clermont et al., 1993; Longo et al., 1987; Oko, 1995; Oko and Sutovsky, 2009; Toshimori, 2003; Tovich et al., 2004). The PT is composed of a variety of cytosolic and nuclear proteins (Dadoune, 2003; Longo et al., 1987; Meistrich, 1993; Oko and Sutovsky, 2009; Toshimori, 2003) which, together with the specific location of the theca, would suggest that it serves to bind the acrosome and post-acrosomal plasmalemma to the nucleus, thereby assisting in maintaining the shape of the sperm head (Longo and Cook, 1991). Accentuating 
this role of the PT is the observation that failure of the PT to form correctly results in the formation of acrosomeless round-headed human sperm (Escalier, 1990). Although material similar in appearance and location to that of the mammalian PT has been described/illustrated during avian spermiogenesis (Aire, 2007, 2014; Fawcett et al., 1971), a PT per se has not been specifically identified in this vertebrate order or its possible function in sperm head shaping explained. Based on the results of the present study it is clear that material similar in location to that of the mammalian PT is present during spermatid differentiation in ratites and can also be divided into a SAL and PAS.

Studies on various mammalian species have demonstrated the involvement of the SAL of the PT in acrosomal biogenesis and subsequent binding of the mature acrosome to the nucleus (Oko, 1995; Oko and Sutovsky, 2009; Sedó et al., 2009; Tovich et al., 2004). It has also been implicated, as part of the cytoskeletal system of the developing spermatid, in conformational changes to the sperm head (Mújica et al., 2003). The latter role has been studied in great detail in rodents (Kierszenbaum et al., 2007) and an integrated action proposed whereby the forming acrosome, acroplaxome (a specialized region of the PT underlying the acrosome [Kierszenbaum et al., 2008]), manchette microtubules and contractile protein arrays (F-actin hoops) in the encompassing Sertoli cells all combine to effect head shaping. This has been referred to as the acrosome-acroplaxome-manchette complex (Kierszenbaum and Tres, 2004; Kierszenbaum et al., 2011; Tres and Kierszenbaum, 2009) which, in addition to its proposed head-shaping role, is also involved in the formation of the centriolar complex and development of the flagellum through a process of intramanchette transport (Kierszenbaum, 2002). In ratites a SAL which morphologically resembles that of man and various mammals (Courtens et al., 1976; Dadoune, 1995) is present throughout spermiogenesis. However, the concentration of intermediate filaments denoting this region as the acroplaxome in rodents 
(Kierszenbaum et al., 2003) is not observed. Whereas in mammals the acrosome covers the anterior two thirds of the nucleus, in non-passerine and sub-oscine birds it is short relative to the length of the nucleus (Jamieson et al., 1995). In ratites it covers approximately $10 \%$ of the nucleus and would therefore have little influence on head shaping in general except for the formation of the nuclear rostrum as described above. Additionally, in ratites the conformational changes to the sperm head take place while the circular manchette is in position unlike the situation in mammals where the longitudinal manchette is involved.

The Sertoli cell cytoplasm surrounding the acrosome in ratites is often patchy but when present displays profiles of smooth endoplasmic reticulum close to the cell membrane. However, the actin filaments which, together with the smooth endoplasmic reticulum form the ectoplasmic specializations (Meistrich, 1993; Toshimori, 2009) essential for the functioning of the acrosome-acroplaxome-manchette complex, are not observed in ratites. Likewise, the tubulobulbar complexes identified in rodents and which may play a role in spermatid head shaping (Kierszenbaum and Tres, 2004) are not obvious during ratite spermiogenesis. In respect of head shaping in ratites, therefore, the role of the Sertoli cell appears to be stabilization of the spermatid head within the seminiferous epithelium through the action of adhering cell junctions. Taking these factors into consideration the function of the SAL would seem to be confined to attachment of the acrosome to the nucleus, enabling it to shape the nuclear rostrum.

The PAS of the mammalian PT is assembled independently from that of the SAL and is composed of various proteins deposited by the microtubules of the LM as it retreats caudally during spermatid elongation (Oko and Sutovsky, 2009). However, in ratite spermatids a fibro-granular layer of material is present between the manchette microtubules and the outer 
nuclear membrane of both the CM and LM. Russell et al. (1991) describe a similar layer of material in early rodent spermatids which extends short rod-like elements (linkers) that link individual microtubules and also connect the microtubules to the nuclear envelope. Based on these observations the authors conclude that "the rod-like linkages of the manchette ensure that the nuclear envelope remains at a constant distance from the manchette microtubule and that this is a major factor acting to impart nuclear shape changes on a region of the head caudal to the acrosome during the early elongation phase of spermiogenesis". The concentration of dynein located between the nuclear surface and the manchette in rats (Yoshida et al., 1994) adds credence to this suggestion. During the duration of the CM in ratites, the PAS displays localized groups of finger-like elaborations that appear to extend between the manchette microtubules and the outer nuclear membrane in similar fashion to the linkers described by Russell et al.(1991). When the LM is formed late in ratite spermiogenesis, the PAS elaborations, although spread throughout the nuclear surface, are concentrated in the region immediately beneath the acrosome. Whether the finger-like elaborations fulfil a similar function to the linkers described in rodent spermatids remains unknown, although they would appear to maintain the spatial relationship of the manchette relative to the nuclear envelope. When the LM is established in ratites, the nucleus has already adopted its final shape (only further compaction of the chromatin is observed thereafter) and it seems likely that the function of the LM and associated PAS is the caudal translocation of cytoplasm to the nuclear base/midpiece of the spermatid prior to spermiation. It is tempting to speculate that the concentration of finger-like projections around the base of the nucleus fulfil a similar function to that of the nuclear ring in mammals by anchoring the proximal end of the LM. The precise alignment and repeatability of the finger-like elaborations of the PAS is suggestive of a mechanical role for this structure. However, the composition of the nuclear and cytosolic proteins making up the avian PAS, as determined 
for mammals (Longo et al., 1987; Meistrich, 1993; Oko, 1995; Tovich et al., 2004; Oko and Sutovsky, 2009) remains to be resolved before a more meaningful conclusion on the role of this structure can be arrived at.

\section{Conclusion}

Head shaping in ratites results from a carefully co-ordinated combination of extrinsic and intrinsic factors. Based on the sequence of morphological events observed during spermiogenesis, it is concluded that the $\mathrm{CM}$ is responsible for the initial transformation in shape of the spermatid nucleus. This is achieved through the progressive tightening of the microtubular coils of the $\mathrm{CM}$, resulting in nuclear elongation. During this process the spatial relationship between the $\mathrm{CM}$ and outer nuclear membrane is maintained and stabilized by localized elaborations of the post-acrosomal PT. Following elongation of the spermatid nucleus the chromatin condenses independently within the confines of the nuclear envelope with the CM merely acting to maintain the shape of the nucleus while this process is underway, to further compress the nuclear envelope, and seemingly orientate the subunits of the condensing chromatin. The acrosome, possibly in conjunction with the condensing karyoplasm, shapes the tip of the nucleus, forming the nuclear rostrum. Unlike the situation in mammals, the surrounding Sertoli cells appear to play a limited role in sperm head shaping, acting only to correctly orientate and stabilize the elongating spermatids within the seminiferous epithelium. The LM assists in the caudal translocation of material during spermatid elongation and also stabilizes the head and midpiece during this process. Material similar in location to that of the mammalian PT is present during spermatid differentiation in ratites and can be divided into a SAL and PAS. This material, by virtue of the formation of finger-like elaborations, seemingly forms an association between the manchette microtubules and the nuclear envelope, and therefore appears to play an important role both in head 
shaping and stabilization. It is also clear that, although a general pattern can be discerned, the combination of factors determining head shaping in vertebrates may differ between species. This study also indicates that ratite sperm, with their relatively uncomplicated design, may prove a useful model for determining the molecular mechanisms and dynamics involved in spermatid head shaping.

\section{Acknowledgements}

The authors wish to thank Ms Estelle Mayhew for the schematic illustration of spermiogenesis.

\section{Funding}

This research did not receive any specific grant from funding agencies in the public, commercial, or not-for-profit sectors.

\section{References}

Aire, T.A. 2003. Ultrastructural study of spermiogenesis in the turkey, Meleagris gallopavo. Br. Poult. Sci. 44, 674-682.

Aire, T.A. 2007. Spermatogenesis and testicular cycles. In: Jamieson, B.M.G. (ed.), Reproductive Biology and Phylogeny of Birds Vol 6A. Science Publishers, Jersey, pp. 279347.

Aire, T.A. 2014. Spermiogenesis in birds. Spermatogenesis 4:3, e959392. 
Barth, A.D., Oko, R.J. 1989. Abnormal morphology of bovine spermatozoa. Iowa State University Press, Ames.

Clermont, Y., Oko, R., Hermo, L. 1993. Cell biology of mammalian spermiogenesis. In: Desjardins, C., Ewing, L.L. (eds.), Cell and molecular biology of the testis. Oxford University Press, New York, pp. 332-376.

Courtens, J.L., Courot, M., Flechon, J.E. 1976. The perinuclear substance of boar, bull, ram and rabbit spermatozoa. J. Ultrastruct. Res. 57, 54-64.

Dadoune, J-P. 1995. The nuclear status of human sperm cells. Micron 26, 323-345.

Dadoune, J-P. 2003. Expression of mammalian spermatozoal nucleoproteins. Microsc. Res. Techniq. 61, 56-75.

Du Plessis, L. 2013. The morphology and development of normal and abnormal spermatozoa in the emu, Dromaius novaehollandiae. University of Pretoria, Pretoria, Thesis.

Du Plessis, L., Soley, J.T. 2012. Abaxial tail implantation in the emu, Dromaius novaehollandiae: morphological characteristics and origin of a rare avian sperm defect. Theriogenology 77, 1137-1143.

Du Plessis, L., Soley, J.T. 2013. A novel transient structure with phylogenetic implications found in ratite spermatids. BMC Evol. Biol. 13,104. 
Du Plessis, L., Soley, J.T. 2014. A re-evaluation of sperm ultrastructure in the emu, Dromaius novaehollandiae. Theriogenology 81, 1073-1084.

Escalier, D. 1990. Failure of differentiation of the nuclear-perinuclear skeletal complex in the round-headed human spermatozoa. Int. J. Dev. Biol. 34, 287-297.

Fawcett, D.W., Anderson, W.A., Phillips, D.M. 1971. Morphogenetic factors influencing the shape of the sperm head. Dev. Biol. 26, 220-251.

Gunawardana, V.K., Scott, M.G.A.D. 1977. Ultrastructural studies on the differentiation of spermatids in the domestic fowl. J. Anat. 124, 741-755.

Hermo, L., Pelletier, R-M., Cyr, D.G., Smith, C.E. 2010. Surfing the wave, cycle, life history, and genes/proteins expressed by testicular germ cells. Part 2: Changes in spermatid organelles associated with development of spermatozoa. Microsc. Res. Tech. 73, 279-319.

Holstein, A.F., Roosen-Runge, E.C. 1981. Atlas of human spermatogenesis. Grosse Verlag, Berlin.

Humphreys, P.N. 1975. The differentiation of the acrosome in the spermatid of the budgerigar (Melopsittacus undulates). Cell Tissue Res. 156, 411-416.

Jamieson, B.G.M. 2007. Avian spermatozoa: structure and phylogeny. In: Jamieson, B.M.G. (ed.), Reproductive Biology and Phylogeny of Birds Vol. 6A. Science Publishers, Jersey, pp. $349-512$. 
Jamieson, B.G.M., Koehler, L., Todd, B.J. 1995. Spermatozoal ultrastructure in three species of parrots (Aves, Psittaciformes) and its phylogenetic implications. Anat. Record 241, 461468.

Jamieson, B.M.G., Tripepi, R. 2005. Ultrastructure of the spermatozoon of Apus apus (Linnaeus 1758), the common swift (Aves: Apodiformes; Appodidae), with phylogenetic implications. Acta Zool., Stockholm 86, 239-244.

Kierszenbaum, A.L. 2002. Intramanchette transport (IMT): managing the making of the spermatid head, centrosome and tail. Mol. Reprod. Dev. 63, 1-4.

Kierszenbaum, A.L., Rivkin, E., Tres, L.L. 2003. Acroplaxome, an F-actin-keratincontaining plate, anchors the acrosome to the nucleus during shaping of the spermatid head. Mol. Biol. Cell 14, 4628-4640.

Kierszenbaum, A.L., Rivkin, E., Tres, L.L. 2007. Molecular biology of sperm head shaping. Soc. Reprod. Fertil. Suppl. 65, 33-43.

Kierszenbaum, A.L., Rivkin, E., Tres, L.L. 2008. Expression of Fer Testis (FerT) tyrosine kinase transcript variants and distribution sites of FerT during the development of the acrosome-acroplaxome-manchette complex in rat spermatids. Dev. Dynam. 237, 3882-3891.

Kierszenbaum, A.L., Rivkin, E., Tres, L.L. 2011. Cytoskeletal track selection during cargo transport in spermatids is relevant to male fertility. Spermatogenesis 1, 221-230. 
Kierszenbaum, A.L., Tres, L.L. 2004. The acrosome-acroplaxome-manchette complex and the shaping of the spermatid head. Arch. Histol. Cytol. 67, 271-284.

Lin, M., Jones, R.C. 1993. Spermiogenesis and spermiation in the Japanese quail (Coturnix coturnix japonica). J. Anat. 183, 525-535.

Longo, F.J., Cook, S. 1991. Formation of the perinuclear theca in spermatozoa of diverse mammalian species: Relationship of the manchette and multiple band polipeptides. Mol. Reprod. Dev. 28, 380-393.

Longo, F.J., Krohne, G., Franke, W.W. 1987. Basic proteins of the perinuclear theca of mammalian spermatozoa and spermatid: A novel class of cytoskeletal elements. J. Cell Biol. $105,1105-1120$.

Lovas, E.M., Filippich, L.J., Johnston, S.D. 2012. Spermiogenesis in the Australian cockatiel Nymphicus hollandicus. J. Morphol. 273, 1291-1305.

Mattei, C., Mattei, X., Manfredi, J-L. 1972. Electron microscopic study of the spermiogenesis of Streptopelia roseogrisea. J. Submicr. Cytol. 4, 57-73.

McIntosh, J.R., Porter, K.R. 1967. Microtubules in the spermatids of the domestic fowl. J. Cell Biol. 35,153-173.

Meistrich, M. 1993. Nuclear morphogenesis during spermiogenesis. In: De Krester DM (ed.), Molecular biology of the male reproductive system. Academic Press, San Diego, pp.67-97. 
Meistrich, M.L., Trostle-Weige, P.K., Russell, L.D. 1990. Abnormal manchette development in spermatids of azh/azh mutant mice. Am. J. Anat. 188, 74-86.

Mújica, A., Navarro-García, F., Hernández-González, E.O., De Lourdes Juárez-Mosqueda, M. 2003. Perinuclear theca during spermatozoa maturation leading to fertilization. Microsc. Res. Tech. 61, 76-87.

Nagano, T. 1962. Observations on the fine structure of the developing spermatid in the domestic chicken. J. Cell Biol. 14, 193-205.

Nozawa, Y.I., Yao, E., Gacayan, R., Xu, S-M., Chuang, P-T. 2014. Mammalian Fused is essential for sperm head shaping and periaxonemal structure formation during spermatogenesis. Dev. Biol. 388, 170-180.

Okamura, F., Nishiyama, H. 1976. The early development of the tail and the transformation of the shape of the nucleus of the spermatid of the domestic fowl, Gallus gallus. Cell Tissue Res. 169, 345-359.

Oko, R., Sutovsky, P. 2009. Biogenesis of sperm perinuclear theca and its role in sperm functional competence and fertilization. J. Reprod. Immunol. 83, 2-7.

Oko, R.J. 1995. Developmental expression and possible role of perinuclear theca proteins in mammalian spermatozoa. Reprod. Fertil. Dev. 7, 777-797. 
Phillips, D.M. 1974. Spermiogenesis. Academic Press, New York.

Phillips, D.M., Asa, C., Stover, J. 1987. Ultrastructure of spermatozoa of the white-naped crane. J. Submicr. Cytol. Path. 19, 489-494.

Phillips, D.M., Asa, C.S. 1989. Development of spermatozoa in the rhea. Anat. Record 223, 276-282.

Ploën, L. 1971. A scheme of rabbit spermateleosis based upon electron microscopic observations. Z. Zellforsch Mik. Ana. 11, 553-564.

Prisant, N., Escalier, D., Soufir, J-C., Morillon, M., Schoevaert, D., Misrahi, M., Tachdjian, G. 2007. Ultrastructural nuclear defects and increased chromosome aneuploidies in spermatozoa with elongated heads. Hum. Reprod. 22, 1052-1059.

Russell, L.D., Russell, J.A., MacGregor, G.R., Meistrich, M.L. 1991. Linkage of manchette microtubules to the nuclear envelope and observations of the role of the manchette in nuclear shaping during spermiogenesis in rodents. Am. J. Anat. 192, 97-120.

Russell, L.D., Ying, L., Overbeek, P.A. 1994. Insertional mutation that causes acrosomal hypo-development: its relationship to sperm head shaping. Anat. Record 238, 437-455.

Saita, A., Longo, O.M., Tripepe, S. 1983. Osservazioni comparative sulla spermiogenesi. III. Aspetti ultrastrutturali della spermiogenesi di Jacana jacana (Caradriformes). Accad. Naz. Lincei Rome 74, 417-430. 
Saita, A., Tripepi, S., Longo, O.M. 1982. Comparative observations on spermiogenesis. II. Nuclear shaping in the absence of a microtubular manchette in the spermatids of the bird Crotophaga ani (Cuculiformes). B. Zool. 49, 115-123.

Samour, J.H., Smith, C.A., Moore, H.D., Markham, J.A. 1986. Semen collection and spermatozoa characteristics in budgerigars (Melopsittacus undulates). Vet. Rec. 118, 397399.

Sedó, C.A., Oko, R., Sutovsky, P., Chemes, H., Rawe, V.Y. 2009. Biogenesis of the sperm head perinuclear theca during human spermiogenesis. Fertil. Steril. 92,14773-1474.

Soley, J.T. 1993. Ultrastructure of ostrich (Struthio camelus) spermatozoa: I. Transmission electron microscopy. Onderstepoort J. Vet. Res. 60, 119-130.

Soley, J.T. 1994. Centriole development and formation of the flagellum during spermiogenesis in the ostrich (Struthio camelus). J. Anat. 185, 301-313.

Soley, J.T. 1996.Differentiation of the acrosomal complex in ostrich (Struthio camelus) spermatids. J. Morphol. 227, 101-111.

Soley, J.T. 1997. Nuclear morphogenesis and the role of the manchette during spermiogenesis in the ostrich (Struthio camelus). J. Anat. 190, 563-576. 
Sperry, A.O. 2012. The dynamic cytoskeleton of the developing male germ cell. Biol. Cell 104, 297-305.

Toshimori, K. 2003. Biology of spermatozoa maturation: An overview with an introduction to this issue. Microsc. Res. Techniq. 61, 1-6.

Toshimori, K. 2009. Dynamics of the mammalian sperm head: modifications and maturation events from spermatogenesis to egg activation. Adv. Anat. Embryol. Cell Biol. 204, 5-94.

Toshimori, K., Ito, C. 2003. Formation and organization of the mammalian sperm head. Arch. Histol. Cytol. 66, 383-396.

Tovich, P.R., Sutovsky, P., Oko, R.J. 2004. Novel aspect of perinuclear theca assembly revealed by immunolocalization of non-nuclear somatic histones during bovine spermiogenesis. Biol. Reprod. 71, 1182-1194.

Tres, L.L., Kierszenbaum, A.L. 2009. The molecular landscape of spermatogonial stem cell renewal, meiotic sex chromosome inactivation, and spermatic head shaping. In: Chedrese, P.J. (ed.), Reproductive Endocrinology: A Molecular Approach. Springer, New York, pp. 313-321.

Tripepi, S., Tavolaro, P., Rossi, F. 1991. The evolution of microtubular organization during spermiogenesis in birds. Selected Symposia and Monographs U.Z.I, 4. In: Ghiara, G. (ed.), Symposium on the Evolution of Terrestrial Verebrates. Mucchi, Modena, pp.631-636. 
Yoshida, T., Ioshii, S.O., Imanaka-Yoshida, K., Izutsu, K. 1994. Association of cytoplasmic dynein with manchette microtubules and spermatid nuclear envelope during spermiogenesis in rats. J. Cell Sci. 107, 625-633.

Zamboni, L. 1987. The ultrastructural pathology of the spermatozoon as a cause of infertility: the role of electron microscopy in the evaluation of semen quality. Fertil. Steril. 48, 711-734.

Zhuang, T., Hess, R.A., Kolla, V., Higashi, M., Raabe, T.D., Brodeur, G.M. 2014. CHD5 is required for spermiogenesis and chromatin condensation. Mech. Develop. 131, 35-46. 